\title{
Lumped-circuit Modeling of Surface Charge Decay in a Needle-plane geometry
}

\author{
X. Wang, N. Taylor, M. Ghaffarian Niasar, R. Clemence Kiiza, H. Edin \\ KTH-Royal Institute of Technology, \\ School of Electrical Engineering, \\ Teknikringen 33, SE-100 44, Stockholm, Sweden
}

\begin{abstract}
Partial discharge (PD) tests were carried out in a needle-plane geometry where the plane was covered by a dielectric barrier. PDs were generated by the application of a periodic negative step voltage. A typical lumped circuit model of the dielectric was created to simulate the bulk and surface conduction of space charge decay processes during corona discharges. The comparison was made between the simulation and the experimental results at the first cycle of the applied voltage. The result shows that at a lower applied voltage, the surface charges decay slower because of a lower surface and bulk conduction, however, they decay faster with the increasing applied voltage due to the increasing conduction.
\end{abstract}

\section{Introduction}

Partial discharge (PD) measurement provides a common tool to diagnose electrical insulation systems infested by defects. PDs are usually measured with 50 $\mathrm{Hz}$ AC sinusoidal voltage stimulus in an on-line situation, but can also be measured at other voltages in off-line situations $[1,2]$.

When a solid dielectric barrier is limiting the discharge activity, every discharge event deposits a certain amount of charges on the surface of the barrier. The deposited charges on the insulating surface will remain for a shorter or longer time depending on the decay mechanism due to different physical origins, such as bulk conduction, surface conduction, diffusion processes and neutralization from charges in the surrounding gas. Charge decay on the insulating surface can be investigated by the measurements or simulation of surface potential [3], charge density [4] and repetition rate of discharge activities [5].

The lumped-circuit model of a dielectric is an engineering method to consider the insulating material as distributed capacitance and resistance [6-9]. One particular feature of this model is that the time dependent behavior of the current flowing on the surface will follow $I(t) \propto t^{-1 / 2} \quad$ [7]. The surface potential decay caused by the ohmic conduction of both the surface and the volume of the sample was investigated in [3]. Therefore, lumped-circuit model could also be a useful method to study the surface charge decay processes including surface and bulk conduction.
In this study, a new method was explored based on a sequence of negative step pulses, of in principle variable duration $T_{1}$, followed by a pause period of variable duration $T_{2}$, with the voltage waveform shown in [5]. The aim is to study how such voltage application affects the relaxation of surface charges on the dielectric surface from previous discharges. The evolution of corona pulses at the different negative step voltage levels of eight consecutive cycles for the case of $T_{1}=100 \mathrm{~ms}$ and $T_{2}=10 \mathrm{~s}$ can be found in [5]. However, this paper mainly focuses on the modeling of surface charge decay of corona discharges based on the lumped-circuit model. By comparing with the experimental results at the first cycle of the applied voltage, the space charge decay processes based on the surface and bulk conduction was investigated.

\section{Experimental setup}

The time-resolved PD measurement system consists of an Agilent 33120A function generator, a TREK 20/20 high-voltage amplifier, a coupling capacitance, a detection impedance, a Yokogawa DL750 Scope Corder and a computer, as shown in Fig. 1.

The test voltage was generated by the function generator and then amplified by the high-voltage amplifier. The coupling capacitance $C_{\mathrm{K}}$ was $250 \mathrm{pF}$ (two $500 \mathrm{pF}$ LCC ceramic capacitors in series), which was in series with the detection impedance $Z_{\mathrm{t}}$ with a resistance of $1 \mathrm{k} \Omega$. The partial discharge pulses were acquired with the Scope Corder, with 12-bit A/D resolution, a sampling rate of $10 \mathrm{MS} / \mathrm{s}$, and a deep memory of $250 \mathrm{MS}$, which allowed long time pulse sequential analysis.

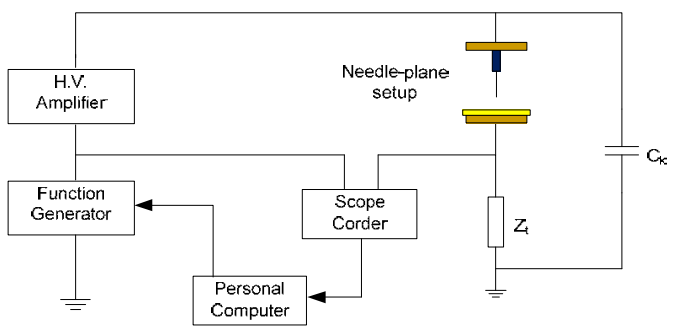

Fig.1 - Schematic of time-resolved PD measuirement system

All the discharges were created as corona pulses in the needle-plane geometry, where a polycarbonate plate was placed on the ground electrode. The dimension of the ground electrode, the needle and the insulating 
sheet used here can be found in [5]. The needle tip has a radius of $15 \mu \mathrm{m}$. The distance between the needle tip to ground electrode was $1 \mathrm{~cm}$. Before each measurement at a new applied voltage, the test object was allowed to relax by grounding the material to remove all the charges on the surface of the material. Air temperature and pressure in the test room were about $21^{\circ} \mathrm{C}, 100 \mathrm{kPa}$, respectively.

\section{Theory}

\subsection{Current-voltage characteristics}

Corona discharge occurs when the applied voltage exceeds the inception voltage. After the corona starts, the corona current $I_{\mathrm{C}}$ then rises with the increasing voltage $V$, following a current-voltage characteristic equation, such as

$$
I_{C}=K\left(V-V_{0}\right)^{2}
$$

where $V_{0}$ is the corona inception voltage, $K$ is a constant. Assume the single discharge pulse does not have a cumulative effect on the background electric field, so every corona discharge event from the needle tip should give almost the same amount of charge $q_{0}$. Therefore, the cumulative corona charge $Q(t)$ during discharge activities is

$$
Q(t)=q_{0} N(t)
$$

where $N(t)$ is the cumulative number of corona pulses from the start. The average corona current $I_{\mathrm{C}}$ is then obtained by

$$
I_{C}=\frac{d Q(t)}{d t}=q_{0} \frac{d N(t)}{d t}
$$

Every corona discharge deposits a certain amount of charges on the dielectric surface, leading to a charge accumulation, and a corresponding increase of the surface potential. The surface potential beneath the needle tip is $V_{\mathrm{P}}$. This causes reduction of the voltage difference across the gap to $V-V_{\mathrm{P}}[10]$, leading to the current-voltage relation with the same constant value of $K$, given as

$$
I_{C}=K\left(V-V_{P}-V_{0}\right)^{2}
$$

As corona charges build up on the insulating surface, $V_{\mathrm{P}}$ increases. Therefore, the corona current $I_{\mathrm{C}}$ decreases, and even becomes zero if $V_{\mathrm{P}}$ builds up high enough. Thus, from equation (3) and (4), the relation between the cumulative number of discharge pulses and the applied voltage can be achieved.

\subsection{Lumped circuit modeling of charge decay}

An equivalent circuit is proposed to describe the surface conduction and bulk properties of dielectric on the plane, seen in Figure 2. Assume the discharge area as a circular region, as well as the dielectric.

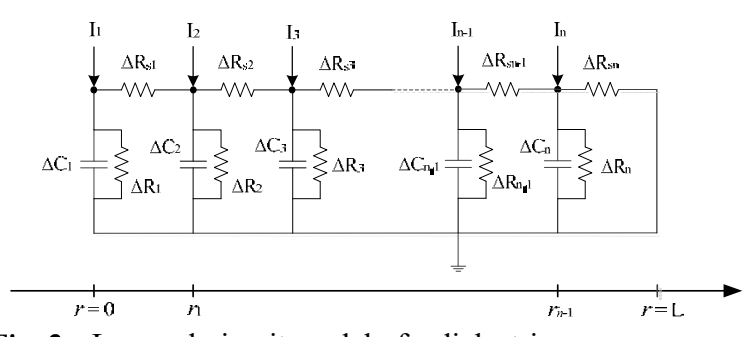

Fig. 2 - Lumped-circuit model of a dielectric

Each ring-shaped element of the bulk has a capacitance and resistance given by

$$
\begin{aligned}
\Delta C_{1} & =\varepsilon_{0} \varepsilon_{r} \pi r_{1}^{2} / d \\
\Delta C_{i} & =\varepsilon_{0} \varepsilon_{r} \pi\left(r_{i}^{2}-r_{i-1}^{2}\right) / d \\
\Delta R_{1} & =\rho d / \pi r_{1}^{2} \\
\Delta R_{i} & =\rho d / \pi\left(r_{i}^{2}-r_{i-1}^{2}\right)
\end{aligned}
$$

Each ring-shaped element of the surface has a resistance given as

$$
\begin{aligned}
& \Delta R_{s 1}=\rho_{s} r_{1} / 2 \pi r_{1} \\
& \Delta R_{s i}=\rho_{s}\left(r_{i}-r_{i-1}\right) / 2 \pi r_{i}
\end{aligned}
$$

where $r_{i}$ is the external radius of the $i$ th ring, $d$ is the thickness of the dielectric, $\rho$ and $\rho_{\mathrm{s}}$ are the bulk and surface resistivity of the dielectric material, respectively. We have the voltage at node $i$

$$
V_{i}=V\left(r_{i}\right)
$$

The current balance at the first node given by

$$
I_{1}=\Delta C_{1} \frac{d V_{1}}{d t}+\frac{V_{1}-V_{2}}{\Delta R_{s 1}}+\frac{V_{1}}{\Delta R_{1}}
$$

and the current at the node $i(1<i \leq n)$ is

$$
I_{i}+\frac{V_{i-1}-V_{i}}{\Delta R_{s i-1}}=\Delta C_{i} \frac{d V_{i}}{d t}+\frac{V_{i}-V_{i+1}}{\Delta R_{s i}}+\frac{V_{i}}{\Delta R_{i}}
$$

where $I_{i}$ is the corona current element at node $i$. At the last node, $n+1$, the resistive layer is assumed to reach ground, therefore, $V_{n+1}=0$. This discrete formulation will yield an equation system of the form with the matrix $M$ and vector $b$

$$
\frac{d}{d t} \bar{V}_{i}=M \bar{V}_{i}+\bar{b}
$$

where the matrix $M$ and vector $b$ can be found in [7]. When corona occurs, the corona current density distribution on a conducting plate can be described by Warburg's relation given as

$$
J_{S}(r)=J_{0} \cos ^{m} \theta
$$

where $J_{0}$ is the current density directly beneath the discharge point. Generally, $m \approx 5$ for $\theta \leq 60^{\circ}$ has been found to be in harmony with the empirical data. It is assumed that the Warburg's relation is even true for the insulating surface, as shown in Figure 3, so that the 
radial dependent current density is considered as the initial distribution on the surface. Then corona current at node $i$ can be given by

$$
I_{i}=I_{C} \frac{3}{2 \pi h^{2}} \frac{h^{5}}{\left(h^{2}+r^{2}\right)^{5 / 2}} \pi\left(r(i+1)^{2}-r(i)^{2}\right)
$$

with the corona current source $I_{\mathrm{C}}$ from equation (4). More details of the derivation process can be found in [5]. A matlab program was made based on equation system (13) to investigate the surface potential $V_{\mathrm{P}}$ and the cumulative number of discharge pulses $N(t)$.

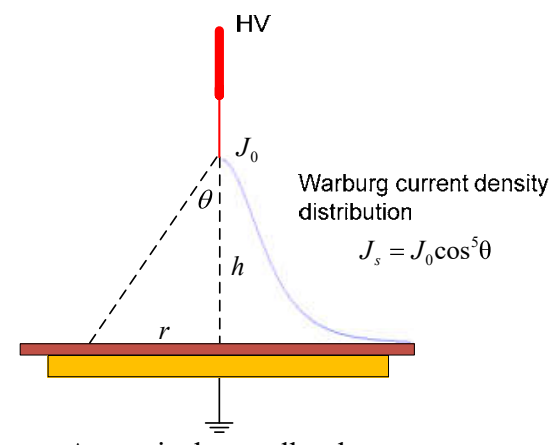

Fig. 3 - A typical needle-plane geometry showing Warburg's relation on insulating plane

\section{Results and discussion}

The simulation results from the lumped circuit model here are compared with the experimental ones at one cycle of the applied voltage based on the cumulative number of corona pulses $N(t)$. From the simulation, three parameters need to be adjusted in order to get a better fitting with the experimental results; they are the bulk and surface resistivity $\rho$ and $\rho_{\mathrm{s}}$, as well as the constant $K$ in equation (4). All the simulations were carried out up to $100 \mathrm{~ms}$ in order to compare with the experimental results.

The corona inception voltage is $V_{0}=3.2 \mathrm{kV}$ in this condition. When the applied voltage is at the lower value of $V=4 \mathrm{kV}$, the behavior of $N(t)$ from the measurement has a good agreement with two simulations, showing exponentially increaseing $N(\mathrm{t})$ with a short rise time of $20 \mathrm{~ms}$, as shown in Fig. 4.

Fig. 5 shows the surface potential behavior over time at a fixed $r$ position on the surface of the dielectric. Every curve corresponds to the surface potential at one specific node in the circuit shown in Fig. 2. It is obvious that the top curve with the highest value indicates the surface potential on the center of the dielectric beneath the needle tip; after that, the curves in sequence show the potential changing along the surface towards the edge, until the bottom one is the potential at the edge of the dielectric, which is set to zero in this simulation. Moreover, almost all the potential curves follow the exponential increasing with the increased time until $20 \mathrm{~ms}$ and then level off. This is because the surface charges deposited on the dielectric surface decay slower due to the higher bulk and surface resistivity, thus more and more charges stay on the surface, leading to an increasing surface potential $V_{\mathrm{p}}$ in a very short time, therefore, $V-V_{\mathrm{p}}$ is decreased, which decelerates the discharge activities. Since Warburg's relation is considered as the initial condition of charges built-up on the dielectric surface in the simulation, shown in Fig. 3, therefore, the potential distribution along the radial direction on the surface always follows the Warburg's relation, with the increasing value of surface potential over time, as shown in Fig. 6.

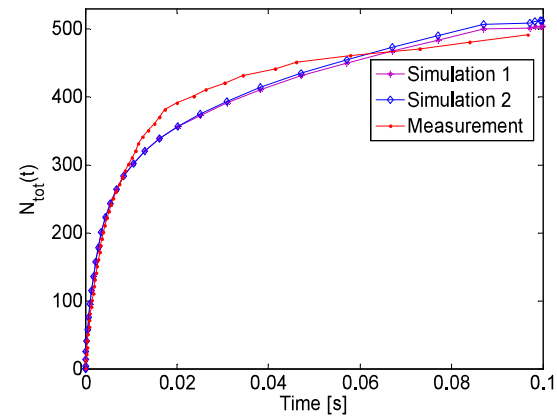

Fig. 4 - The comparison of the cumulative number of corona pulses at $V=4 \mathrm{kV}$ with the parameters of $K=7.2 \times 10^{-12}$ $\left[C /\left(V^{2} \times s\right)\right], \rho_{\mathrm{s}}=10^{11} \Omega, \rho=10^{12} \Omega \cdot \mathrm{m}$ in simulation 1 and $\rho$ $=10^{11} \Omega \cdot \mathrm{m}$ in simulation 2 .

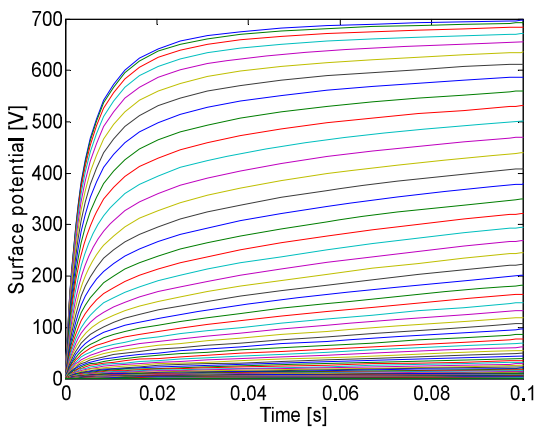

Fig. 5 - The surface potential behavior over time at fixed $r$ position on the surface of the dielectric at $V=4 \mathrm{kV}$ with the parameters of simulation 1 in Fig. 4. The total number of nodes is $n=100$.

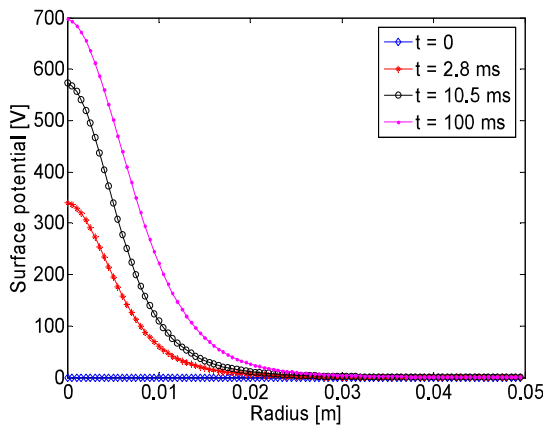

Fig. 6 - The surface potential behavior over time along the radial direction on the surface at $V=4 \mathrm{kV}$ with the parameters of simulation 1 in Fig. 4.

As the applied voltage is increased, the cumulative number of corona pulses $N(t)$ behaves differently compared with the case of a lower applied voltage. The results in Fig. 7 show a slower exponentially increasing behavior, and the same to the surface potential behavior in Fig. 8. The potential $V_{\mathrm{p}}$ is higher 
than before because of the higher applied voltage. The rise time of the curves here is longer because of the lower value of constant $K$, which controls the corona current going to the dielectric.

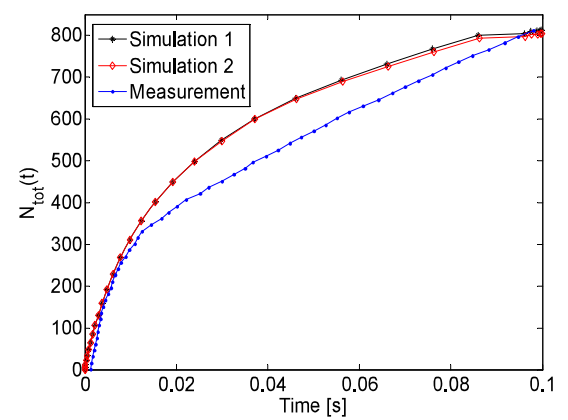

Fig. 7 - The comparison of the cumulative number of corona pulses at $V=4.8 \mathrm{kV}$ with the parameters of $K=0.8 \times 10^{-12}$ $\left[C /\left(V^{2} \times s\right)\right], \rho_{\mathrm{s}}=10^{11} \Omega, \rho=10^{11} \Omega \cdot \mathrm{m}$ in simulation 1 and $\rho$ $=10^{12} \Omega \cdot \mathrm{m}$ in simulation 2 .

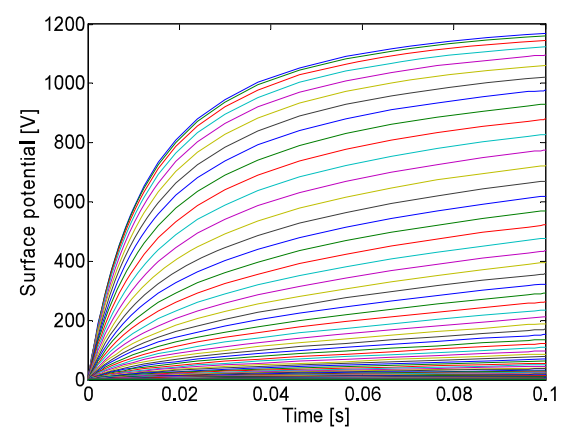

Fig. 8 - The surface potential behavior over time at fixed $r$ position on the surface of the dielectric at $V=5.4 \mathrm{kV}$ with the parameters of simulation 2 in Fig. 8. The total number of nodes is $n=100$.

More comparisons for the cases of higher applied voltage levels at $V=5.4 \mathrm{kV}$ and $V=6 \mathrm{kV}$ are shown in Fig. 9. It can be observed that the cumulative number of corona pulses $N(t)$ increases almost linearly from the start, and then reach to a very high value. This is because the space charges depostied on the dielectric decay faster due to the higer bulk and surface conduction, leading to a very small increase of $V_{\mathrm{p}}$ compared to the applied voltage, therefore, $V-V_{\mathrm{p}}$ is big enough to cause a constant repetition rate of discharge activities.

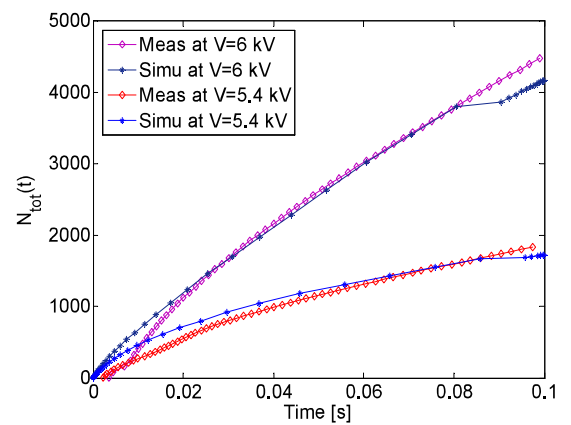

Fig. 9 - The comparison of the cumulative number of corona pulses for the case of $V=5.4 \mathrm{kV}$ with the parameters of $K=$ $0.6 \times 10^{-12}\left[C /\left(V^{2} \times s\right)\right], \rho_{\mathrm{s}}=2 \times 10^{10} \Omega$ and $\rho=10^{10} \Omega \cdot \mathrm{m}$, for the case of $V=6 \mathrm{kV}$ with the parameters of $K=0.5 \times 10^{-12}$ $\left[C /\left(V^{2} \times s\right)\right], \rho_{\mathrm{s}}=5 \times 10^{9} \Omega$ and $\rho=10^{9} \Omega \cdot \mathrm{m}$.

\section{Conclusion}

A lumped-circuit model was proposed to simulate the surface and bulk conduction for the surface charge decay processes of corona discharge. Simulations were compared with experimental results acquired from the needle-plane corona setup after the applied step voltages. Two important parameters from the model have been investigated here, that is the cumulative number of corona pulses $N(t)$ and surface potential on the dielectric $V_{\mathrm{p}}$.

The results indicate that the dielectric covering on the surface of ground electrode strongly affect the discharge activities by cumulating charges on it. Thus corona discharge activities at the different applied voltage levels are quite different due to the charges building on the surface of dielectric, and this built-up surface potential is controlled by the surface and bulk conduction in this model. Therefore, at a lower applied voltage, the surface charges decay slower because of a lower surface and bulk conduction, however, they decay faster with the increasing applied voltage due to the increasing conduction.

\section{References}

[1] D. Fabiani, G. C. Montanari, A. Cavallini, and G. Mazzanti, "Relation between space charge accumulation and partial discharge activity in enameled wires under PWM-like voltage waveforms," IEEE Transactions on Dielectrics and Electrical Insulation, Vol. 11, No. 3, pp. 393-405, 2004.

[2] K Wu, T. Okamoto, and Y. Suzuoki, "Effects of discharge area and surface conductivity on partial discharge behavior in voids under square voltages," IEEE Transactions on Dielectrics and Electrical Insulation, Vol. 14, No. 2, pp.461-470, 2007.

[3] A. Crisci, "Surface-potential decay due to surface conduction", Eur. Phys. J. AP, Vol. 4, pp. 107-116, 1998.

[4] G. Chen, "A new model for surface potential decay of corona-charged polymers", J. Phys. D: App. Phys., Vol. 43, pp. 1-7, 2010.

[5] X. Wang, "Partial discharge analysis at arbitrary voltage waveform stimulus", Licentiate thesis, KTH, Stockholm, Sweden, 2012.

[6] K. Wu, T. Ijichi, T. Kato, Y. Suzuoki, F. Komori, and T. Okamoto, "Contribution of surface conductivity to the current forms of partial discharges in voids," IEEE Transactions on Dielectrics and Electrical Insulation, Vol. 12, No. 6, pp.1116-1124, 2005.

[7] P. A. Heimann, J. E. Olsen, "A sensitive method for measuring surface conductivity of insulators", J. Appl. Phys., Vol. 53, No. 1, pp. 546-549, 1983.

[8] T. C. Chapman, H. J. Wintle, "Dielectric absorption currents and surface charge on polymeric insulators", $J$. Appl. Phys., Vol. 51, No. 9, pp. 4898-4904, 1980.

[9] L. Lachish, I. T. Steinberger, "Electrical current measurements on polystyrene films", J. Phys. D: App. Phys., Vol. 7, pp. 58-68, 1974.

[10] J. A. Cross, "Electrostatics: principles, problems and applications", Adam Hilger, Bristol, UK, 1987. 